\title{
Migraciones y relaciones de poder en la agricultura global contemporánea: entre actualidad y ruptura de la herencia colonial
}

\author{
Gennaro Avallone*
}

\section{Resumen}

El artículo siguiente sostiene dos hipótesis. Por un lado, la existencia de una tendencia a la naturalización de las condiciones de explotación de los trabajadores migrantes en la agricultura contemporánea como un hecho relacionado con la herencia colonial de dominación. Y por el otro lado, se propone reconocer la presencia de las tendencias que cuestionan este proceso de naturalización, ellas también conectadas con el pasado colonial.

El análisis se desarrolla en seis apartados. En el primer apartado se propone el estudio de algunos ejemplos de enclaves agrícolas caracterizados por la inserción de mano de obra migrante. En los apartados dos y tres, el análisis evidencia algunas características estructurales de las vigentes relaciones sociales y de poder en la agricultura global.

En los apartados cuatro y cinco, se pone en evidencia la originalidad del espacio político postcolonial, en el que los sujetos fuera del espacio político como los migrantes pueden convertirse en actores de transformación política y social.

En el apartado seis se presentan las conclusiones del análisis realizado, mostrando la posibilidad de actualizar el lado conflictivo de la herencia colonial, transformando el espacio político postcolonial a través de movimientos sociales heréticos orientados a producir una ruptura del orden político fundando en el principio de separación entre nacionales y no nacionales.

\section{Palabras clave}

Agricultura; Luchas sociales; Colonialidad; Pensamiento de estado; Migraciones.

\section{TITLE}

Migration and Power Relations in the Global Contemporary Agriculture: Between Persistence and Rupture of the Colonial Legacy

\begin{abstract}
The following article develops two hypotheses. On one hand, it puts forward a link between the naturalization of the exploitative conditions of migrant farm workers in contemporary agriculture and the colonial heritage of domination. On the other hand, it proposes to recognize the trends that question this process of naturalization, highlighting that they are also connected to this colonial heritage.
\end{abstract}

The analysis is articulated in six sections. In the first, some agricultural enclaves characterized by an important presence of migrant manpower are analysed. The second and third sections show some structural characteristics of the current social relations of production in agriculture.

In the fourth and fifth sections, the originality of the postcolonial political space is recognized, in which subjects outside the political space, such as migrants, can become important actors of political and social transformation.

Finally, in the sixth section, some conclusions are presented showing the possibility to update the conflictual side of colonial heritage, transforming the postcolonial political space through a rupture of a political order based on the principle of separation between nationals and non-nationals.

\section{KEYWORDS}

Agriculture; Social Struggles; Coloniality; State Thought; Migration. 


\section{ntroducción}

El artículo siguiente sostiene dos hipótesis. Por un lado, la existencia de una tendencia a la naturalización de las condiciones de explotación de los trabajadores migrantes en la agricultura postcolonial contemporánea como un hecho estrictamente relacionado con la herencia colonial de la dominación entre colonizados y colonizadores. Y por el otro lado, se propone reconocer la presencia de sus contra tendencias, es decir, aquellas que cuestionan este proceso de naturalización. Ambas dimensiones están conectadas con el pasado colonial, siendo herederas de relaciones coloniales que siguen activas a pesar de la conclusión formal de las administraciones coloniales.

El crecimiento continuo de la mano de obra migrante en la agricultura capitalista global se ha convertido en un hecho estructural en los últimos 30 años. Un fenómeno que, no lo olvidemos, se realiza en un mundo postcolonial, en el sentido de que estas migraciones están influenciadas por la ambivalencia de la herencia colonial, caracterizada en sí misma por la co-presencia de dos tendencias: una hacia la confirmación de las relaciones de dominación y la otra hacia su cuestionamiento y, entonces, la liberación y la construcción de otras formas de relación y poder ${ }^{1}$.

La primera tendencia se manifiesta en diferentes formas que reproducen la forma colonial de gobierno de las poblaciones y se fundan en la separación jerárquica entre dominantes y dominados, de manera similar - aunque no igual- a la realizada en los contextos que separaban oficialmente y desde el punto de vista legislativo los colonizados de los colonizadores. La segunda tendencia se observa en los mismos movimientos migratorios y en sus capacidades de construir relaciones sociales de poder diferentes de las heredadas del pasado colonial.

El análisis aquí propuesto se desarrolla en seis apartados. En el primer apartado se propone el estudio de algunos ejemplos de enclaves de la agricultura global caracterizados por diferentes formas de inserción de mano de obra migrante interna o internacional. En los apartados dos y tres, el análisis se desarrolla a partir de una experiencia de investigación con trabajadores migrantes en la agricultura italiana evidenciando aquí algunas características de las relaciones sociales y de poder características de los contextos coloniales que han ido más allá de la historia de las administraciones coloniales. Combinando la investigación etnográfica con el análisis de una parte de la literatura teórica desarrollada en el marco de los estudios postcoloniales y decoloniales se reconocerá que la separación jerárquica -característica fundamental de la colonia- aún influencia el presente, y se articula a través de tres principios de organización: la homogeneización de los grupos sociales y raciales, el confinamiento y la raza.

En el apartado cuatro, se pondrá en evidencia la originalidad del espacio político postcolonial, en el que la persistencia de relaciones de separación entre las poblaciones de las áreas centrales y las de las ex áreas coloniales ha sido actualizada tras las victoriosas luchas anticoloniales. Estas luchas han mostrado que los confines no son definitivos y los sujetos

\footnotetext{
1 MEZZADRA, Sandro, "Introducción" en MEZZADRA, Sandro (coord.), Estudios postcoloniales. Ensayos fundamentales, Traficantes de Sueños, Madrid, 2008, pp. 15-33.
} 
fuera del espacio político pueden convertirse en los principales actores de transformación política y social. Como se muestra en el apartado cinco, esta es también la condición de los migrantes, sujetos definidos como extraños a la comunidad, que desafían cotidianamente los confines de la separación mediante la toma de la palabra y a través de las alianzas mestizas con pobladores locales, que están construyendo un mundo herético de relaciones entre humanos, es decir, según el análisis de Abdelmalek Sayad ${ }^{2}$, un mundo que cuestiona la separación naturalizada entra nacionales y no nacionales.

En el apartado seis se presentan las conclusiones del análisis realizado, mostrando la posibilidad de actualizar el lado conflictivo de la herencia colonial, transformando el espacio político postcolonial a través de una ruptura del principio de separación entre nacionales y no nacionales, es decir, entre dominadores y dominados.

\section{Agricultura global y migraciones}

La agricultura global está caracterizada por la inserción permanente de trabajadores inmigrantes internos e internacionales. Son muchos los casos que en los diferentes continentes ponen en evidencia este fenómeno. Cuando el enfoque privilegiado es la organización de los enclaves agrícolas desde el punto de vista de la sociedad, es decir el estudio de la manera de construir las relaciones sociales de producción y su funcionamiento y, por tanto, la tensión entre la reproducción de la vida cotidiana y la reproducción ampliada del capital, el fenómeno tiende a manifestarse con más fuerza. De esta manera, concentrarse en el secreto laboratorio de la producción capitalista de fruta fresca y hortalizas, abandonando la ruidosa y superficial esfera de la circulación, permite conocer los lugares donde se encuentran el capital y la fuerza de trabajo y, por ende, donde se generan el valor y la plusvalía. Ello remite a las palabras de Karl Marx en el capítulo cuarto del primer tomo de El Capital, donde sugiere un descenso "hacia la oculta sede de la producción, en cuyo dintel se lee: (...) Prohibida la entrada salvo por negocios"3.

Observando algunos casos, por ejemplo, los indagados a través de las investigaciones conducidas en el marco del proyecto Enclave ${ }^{4}$ en diferentes enclaves agrícolas en España, Argentina, Uruguay, México y Brasil, se pone en evidencia que, a pesar de las diferencias geográficas, históricas y sociales de estas áreas, las condiciones de trabajo son increíblemente similares en todas ellas. El trabajo está generalmente caracterizado por su extrema flexibilidad y precariedad, salarios bajos y subordinación a las necesidades de las empresas agrícolas. Este dominio se realiza a través de la combinación variable de cinco principales modalidades: la incertidumbre ocupacional y el consecuente miedo al paro; la reproducción del ejército industrial de reserva, mediante la gestión de la mecanización y de la movilidad espacial de la fuerza de trabajo; la intermediación de los contratistas y capataces, que desestructura e individualiza las relaciones laborales, la discriminación y segmentación basadas en la pertenencia nacional y de género, el manejo ambivalente de los estándares de calidad y

\footnotetext{
2 SAYAD, Abdelmalek, La doble ausencia. De las ilusiones del emigrado, a los padecimientos del inmigrado, Anthropos, Barcelona, 2010.

3 MARX, Karl, El capital. Volumen 1, Siglo XXI, Buenos Aires, 2002, p. 123

4 Informaciones sobre el proyecto Enclave se pueden encontrar en el blog:

http://sociologiaruralydelaagricultura.blogspot.com.es.
} 
responsabilidad 5 .

La asimetría de poder entre las empresas y los trabajadores y las trabajadoras es fuerte y ha producido, en la nueva fase de la agricultura transnacional neoliberal, un proceso de desdemocratización de las relaciones laborales, caracterizado por el debilitamiento del trabajo y de los trabajadores, que "tienen mayores dificultades para acceder a los derechos sociales vinculados al trabajo y carecen de un mínimo grado de control sobre sus condiciones de trabajo"6.

Estas condiciones son características de una multiplicidad de enclaves y áreas agrícolas en otras regiones a nivel global?. En todas ellas, el caso californiano marca el estándar de producción en un modelo donde el trabajo es realizado casi exclusivamente por extranjeros, pues son el único recurso controlable por los empresarios ${ }^{8}$. A modo de ejemplo pueden resaltarse los casos de Inglaterra9 ${ }^{\circ}$ Grecia, donde a lo largo de los últimos 25 años la inserción de mano de obra extranjera, que vive y trabaja en condiciones de fuerte explotación sobre todo en algunos enclaves, como en el de Manolada ha crecido exponencialmente ${ }^{10}$. El caso italiano también ha sido largamente estudiado ${ }^{11}$, así como otros contextos de Europa del sur ${ }^{12}$, confirmando que el papel de los migrantes en la agricultura contemporánea es determinante. Canadá13 y América central ${ }^{14}$ son otras dos regiones donde este fenómeno

5 PEDREÑO CÁNOVAS, Andrés, "Encadenados a fetiches. Del enfoque de las cadenas de mercancías a la sosteniblidad social de los enclaves de producción de la 'uva global'" en PEDREÑO CÁNOVAS, Andrés (coord.), De cadenas, migrantes y jornaleros, Talasa, Madrid, 2014, pp. 13-37.

6 DE CASTRO, Carlos, "La desdemocratización de las relaciones laborales en los enclaves globales de producción agrícola" en PEDREÑO CÁNOVAS, Andrés (coord.), De cadenas..., op.cit., p. 59.

7 BONANNO, Alessandro y BARBOSA CAVALCANTI, Josefa Salete, "Introduction" en BONNANNO, Alessandro y BARBOSA CAVALCANTI, Josefa Salete (eds.), Labor Relations in Globalized Food (Research in Rural Sociology and Development, Volume 20), Emerald Group Publishing Limited, Bingley, 2014, pp. 13-49; GERTEL, Jörg y SIPPEL, Sarah Ruth (eds.), Seasonal Workers in Mediterranean Agriculture. The social costs of eating fresh, Routledge, Londres, 2014.

8 MARTIN, Philip, California Hired Farm Labor 1960-2010: Change and Continuity, University of California, 30 de abril de 2011, p. 5: https://migrationfiles.ucdavis.edu/uploads/cf/files/2011-may/martin-california-hired-farmlabor.pdf [Consultado el 23 de julio de 2017].

9 ROGALY, Ben, "Migrant Workers in the Ilo's "Global Alliance against Forced Labour". Report: A Critical Appraisal" en Third World Quarterly, Vol. 29, No 7, 2008, pp. 1431-1447; FRANCES, Jennifer et.al., Temporary workers in UK agriculture and horticulture: a study of employment practices in the agriculture and horticulture industries and co-located packhouse and primary food processing sectors, Precision Prospecting for the Department of Environment, Food and Rural Affairs (DEFRA), Framlingham, Suffolk, 2005.

${ }^{10}$ KASIMIS, Charalambos et.al., "The Precarious Status of Migrant Labour in Greece: Evidence from Rural Areas" en DELLA PORTA Donatella et.al. (coord.), The New Social Division Making and Unmaking Precariousness, Palgrave Macmillan, Basingstoke, 2015, pp. 101-120; PAPADOPOULOS, Apostolos y FRATSEA, Loukia-Maria, "Migrant labour and intensive production in Greece" en CORRADO, Alessandra et.al. (coord.), Migration and agriculture. Mobility and change in the Mediterranean area, Routledge, Nueva York-Londres, 2016, pp. 128-143.

${ }^{11}$ PERROTTA, Domenico y SACCHETTO, Devi, "Migrant Farmworkers in Southern Italy: Ghettoes, caporalato and collective action" en Workers of the World: International Journal on Strikes and Social Conflicts, Vol. 1, No 5, 2014, pp. 75-98; COLLOCA, Carlo y CORRADO, Alessandra (coord.), La globalizzazione delle champagne. Migranti e società rurali nel Sud Italia, FrancoAngelli, Milán, 2012.

12 AVALLONE, GENNARO, "Migraciones y agricultura en Europa del Sur: Emergencia de un nuevo proletariado internacional" en Migraciones Internacionales, Vol. 7, No 4, 2014, pp. 137-169.

${ }^{13}$ CASTRACANI, Lucio, "Étranger(s) au travail. Notes ethnographiques" en Altérités, Vol. 9, No 1, 2016, pp. 4355.

${ }^{14}$ GARCÍA, Martha y DÉCOSSE, Frédéric, "Agricultura intensiva y políticas de migración laboral: Jornaleros centroamericanos en México y marroquíes en Francia" en Migración y desarrollo, Vol. 12, No 23, 2014 , pp. 41-67; ORTIZ, Celso, "Migración, precariedad y sindicalización en la agricultura globalizada" en La Jornada del campo, No 94, 2015: http://www.jornada.unam.mx/2015/07/18/cam-migracion.html [Consultado el 11 de abril de 2017]. 
cobra mucha fuerza: siendo los migrantes la fuente principal de fuerza de trabajo barata, funcional a la reproducción de las condiciones favorable a la producción de comida barata por lo menos para una parte de la población mundial.

Los campos agrícolas a nivel global confirman que las relaciones de poder específicas donde la población migrante juega el rol de los predestinados, es decir, de los que tienen que trabajar en las condiciones impuestas y sin poder cambiar nada, son la norma. Las personas migrantes se encuentran naturalmente en esta posición subalterna, difícilmente cuestionada por las sociedades locales: ellas están en su lugar, el de los explotados, así como las hojas están en su lugar, sobre los árboles. En el siguiente apartado se cuestionará esta naturalización, buscando algunas condiciones de su reproducción y, al mismo tiempo, algunos factores para su superación.

\section{La actualidad de la experiencia colonial en agricultura}

En el mes de Agosto de 2011 participé, en el marco de una experiencia de investigación etnográfica en algunos campos del Sur de Italia, en una asamblea de trabajadores migrantes casi todos procedentes de países del África subsahariana y de piel negra en el así llamado Gran Ghetto de Rignano Garganico en Pullia. Desde hace muchos años se concentran en este sitio cientos de trabajadores que realizan la recogida de los tomates utilizados en la producción de las conservas, contribuyendo así de manera fundamental a la producción de una de las mercancías más conocidas del made in Italy.

La asamblea se formó algunas semanas después de la primera huelga autorganizada en Italia por trabajadores migrantes en agricultura. La huelga tuvo lugar en Nardó, un municipio cercano a la provincia de Lecce, en el mes de Julio de ese año y tuvo como objetivo protestar contra la reducción del salario a destajo que los empresarios agrícolas productores de tomates habían decidido de manera unilateral, pasando a pagar alrededor de 3,50 euros por la recogida de un cajón de 350 kilos $^{15}$.

El tema central de la asamblea fue la cuestión de los derechos de los trabajadores. Los migrantes hablaban de condiciones de empleo, tareas, salarios y derechos en un contexto productivo en el que la división del trabajo corresponde a la división racial. Los negros son la mano de obra, porque son los que trabajan en el campo u organizan la distribución territorial de la fuerza de trabajo como "furgoneteros" e intermediarios informales -caporali-, mientras que los blancos son los que compran la fuerza de trabajo en calidad de empresarios o representantes de estos.

De manera explícita, durante la asamblea, las cuestiones referentes a las relaciones entre trabajadores y empresarios así como los derechos laborales fueron definidos por los propios trabajadores como determinadas por la raza y la jerarquía entra blancos y negros. Durante el encuentro, los participantes evidenciaron la persistencia de condiciones laborales y de vida similares a las vigentes durante el esclavismo, un enemigo de un pasado todavía actual en las relaciones sociales, simbólicas y de poder, aunque no desde el punto de vista

\footnotetext{
${ }^{15}$ Brigate di solidarietà attiva et.al., Sulla pelle viva. Nardò: la lotta autorganizzata dei braccianti immigrati,
} DeriveApprodi, Roma, 2012. 
formal y legislativo. Un hecho que, sin embargo, y pese a su reconversión, ha sido vencido, a lo largo de la historia, a través de las luchas antiesclavistas y anticoloniales.

De hecho, durante la asamblea más de un migrante recordó que esta modalidad de explotación actualizada en el presente puede cambiarse a través de la lucha de los trabajadores, en parte porque ya ha sucedido: es decir, si el pasado de las relaciones de dominación se actualiza en el presente, también se sabe que su derrota es también posible, actualizando las luchas como ya sucedió en el pasado colonial.

Esta manera de hablar de la esclavitud es típicamente postcolonial porque pone en evidencia, por un lado, la fuerza de un pasado que no pasa totalmente, actualizándose con modalidades renovadas en el presente, y, por el otro lado, la fuerza victoriosa de la lucha contra la esclavitud, hija del dominio colonial. Al mismo tiempo, la referencia a la esclavitud tiene un carácter postcolonial pues se realiza en un área históricamente central, el área de la metrópoli.

Lo que se plantea es que un hecho típicamente colonial, el de la esclavitud de los negros, puede ir más allá de los confines establecidos por la geografía centro-periferia y convertirse en una realidad generalizada, si bien diferente respecto al pasado.

En el tiempo postcolonial esto significa que las condiciones de trabajo y vida de una parte de la población migrante, sobre todo de los migrantes de las ex colonias, no son un conjunto de condiciones anacrónicas residuales del pasado, sino que son condiciones actuales que representan una anticipación de lo que puede pasar a una parte de la población que vive en las ex metrópolis.

Los diferentes tiempos experimentados por el mundo colonial, así como el mundo postcolonial, pueden marcar un estadio previo desde el punto de vista histórico y también teórico-sistémico a las condiciones que experimenten los ciudadanos de las ex metrópolis.

En términos históricos, ya ha sido estudiada la relación temporal que se ha construido, por un lado, entre la colonización y el sistema industrial, y, por el otro, entre la colonización y las modalidades de apropiación de la naturaleza extra-humana. En síntesis, se ha visto que la colonia ha sido en muchos aspectos una anticipación, es decir un laboratorio de la modernidad $^{16}$, especialmente un laboratorio de las modalidades de explotación del trabajo vivo y de la apropiación de la naturaleza extra-humana. Por ejemplo, Sandro Mezzadra ha evidenciado que los análisis de Sidney W. Mintz muestran como la plantación de caña de azúcar en las Indias occidentales entre los siglos dieciséis y diecisiete está en el origen "del sistema de fábrica moderno"17. Al mismo tiempo, los análisis de Jason W. Moore sobre la ecología-mundo han confirmado que la colonia no sólo ha anticipado la modernidad capitalista

${ }^{16}$ STOLER, Ann Laura y COOPER, Frederick, "Between Metropole and Colony. Rethinking a Research Agenda" en STOLER, Ann Laura y COOPER, Frederick (coord.), Transitions of Empire. Colonial Cultures in a Bourgeois World, University of California Press, Berkeley, 1997, pp. 1-56; WRIGHT, Gwendolyn, "Tradition in the Service of Modernity: Architecture and Urbanism in French Colonial Policy" en Journal of Modern History, No 59, 1987, pp. 291-316.

${ }^{17}$ MEZZADRA, Sandro, "Introducción ..., op.cit., pp. 15-33. 
sino también ha sido fundamental para su desarrollo ${ }^{18}$. Los procesos de apropiación de los recursos naturales humanos y no humanos de las colonias han sido determinantes para el desarrollo de la acumulación originaria, como en el caso de Potosí en Bolivia, "el excelso monte de plata" que, en el siglo XVI, "para el sabio Rey [...] conquistará al mundo entero"19. La colonia ha sido "la 'otra cara', la alteridad de la modernidad"20: un elemento constitutivo del capitalismo y no un fenómeno secundario o marginal.

La colonia también ha sido un lugar de conflicto y un lugar que ha sido superado por la lucha anticolonial. El conflicto social y político ha caracterizado la vida de las administraciones coloniales, yendo del exitoso caso histórico de la revuelta de los esclavos en Haití a las rebeliones reprimidas con violencia, como en la India por parte de Inglaterra. La colonia también ha sido un laboratorio de liberación y el caso de la revuelta de los esclavos de Haití que en el año 1791 abrogó la esclavitud mucho tiempo antes que otros países "modernos"21 pone bien en evidencia como desde aquí no solo se pueden anticipar la futuras modalidades de explotación y dominación sino también las futuras rutas de emancipación.

La colonia ha sido una experiencia de dominación y también de resistencia y liberación, como muestra la larga historia de luchas anticoloniales. A través de estas luchas, los colonizados se han insertado en el espacio público, tomando la palabra y afirmado su presencia, transformándose en sujetos políticos más allá de las clasificaciones y de los lugares asignados a ellos por los colonizadores.

La colonia ha nacido como frontera de los países centrales y ha sido interpretada como una periferia caracterizada por derechos diferenciados, en una situación "aceptada como parte de una realidad dada por los mismos pensadores que proclamaban que la libertad era el estado natural del hombre y su derecho inalienable"22. A lo largo del tiempo, la colonia se ha convertido en un espacio y, sobre todo, en una experiencia social y de poder determinante en las mismas áreas centrales, en la metrópoli. La producción de la colonia también ha sido constitutiva de las relaciones sociales y de poder en las áreas centrales. En otras palabras, la colonia no ha tenido un límite territorial determinado: por ejemplo, en el análisis de Aime Césaire ${ }^{23}$ se pone de relieve que, durante el nazismo, los dispositivos de represión y gobierno autoritario propios de las colonias se han actualizado en el espacio metropolitano, definiendo una temporalidad postcolonial. Césaire escribió que Hitler y el hitlerismo han perpetrado el "crimen contra el hombre blanco aplica[n]do en Europa procedimientos colonialistas que hasta ahora solo concernían a los árabes de Argelia, a los coolies de la India y a los negros

18 MOORE, Jason W., Capitalism in the web of life: ecology and the accumulation of capital, Verso, Londres y Nueva York, 2015.

19 MOORE, Jason W., "'This lofty mountain of silver could conquer the whole world': Potosí and the political ecology of underdevelopment, 1545-1800" en The Journal of Philosophical Economics, Vol. IV, No 1, 2010, p. 64.

20 DUSSEL, Enrique, "Eurocentrismo y modernidad (Introducción a las lecturas de Frankfurt)" en MIGNOLO, Walter (Coord.), Capitalismo y geopolítica del conocimiento: el eurocentrismo y la filosofía de la liberación en el debate intelectual contemporáneo, Signo, Buenos Aires, 2001, p. 68.

21 JAMES, Cyril Lionel Robert, Los jacobinos negros. Toussaint L'Ouverture y la Revolución de Haití, Fondo de Cultura Económica, México, 2003.

22 BUCK-MORSS, Susan, Hegel y Haiti. La dialéctica amo-esclavo: una interpretación revolucionaria, Grupo Editorial Norma, Buenos Aires, 2005, p. 8.

${ }^{23}$ CÉSAIRE, Aimé, Discurso sobre el colonialismo, Editorial Akal, Madrid, 2006. 
de África"24 tal y como señala Ramón Grosfoguel: "los métodos que históricamente fueron y siguen siendo usados contra el mundo no europeo son inherentes al lado oscuro de la modernidad, es decir, la colonialidad. Antes de ser sus víctimas, los europeos fueron en primer lugar cómplices del nazismo al legitimarlo por siglos siempre que se tratara de poblaciones no europeas. No hay nada original en el nazismo que no fuera antes implementado por el colonialismo contra pueblos no europeos" 25 .

En este sentido vivimos en tiempos postcoloniales, porque, como han reconocido Sandro Mezzadra y Federico Rahola, comentando el análisis de Césaire, "en el momento mismo en que los dispositifs de dominación, en un origen fraguados en el contexto de la experiencia colonial, se infiltran en los espacios metropolitanos, nos encontramos ya, de algún modo, en una época postcolonial" 26 .

La colonia ha sido, entonces, una frontera. Desde el punto de vista del análisis del sistema-mundo 27 , la colonia ha sido fundamental para la apropiación de la naturaleza humana y extra-humana a bajo coste, convirtiéndose en un hecho global constitutivo del capitalismomundo. De hecho, la búsqueda de nuevas fronteras es una característica fundamental del capitalismo, que necesita continuamente a la naturaleza humana y extra-humana barata para alimentar el proceso de acumulación, como ha desarrollado la teoría de la ecología-mundo28. La frontera puede ser horizontal, a través de la conquista imperialista de nuevos territorios, 0 vertical, a través de la conquista de nuevas fuentes de recursos naturales.

La búsqueda constante de nuevas fronteras confirma la persistencia de la lógica del saqueo como ya explicó Marx en el capítulo 24 del primer volumen de El Capital29. Así, como las modalidades de gobierno de la experiencia histórica de la acumulación originaria, basadas en la violencia, la desposesión de los bienes comunes y públicos ${ }^{30}$ y la apropiación barata de los bienes libres de la naturaleza humana y extra-humana ${ }^{31}$ no son más que una continuación de estos procesos.

La búsqueda continua de nuevas fronteras de bienes es constitutiva del capitalismo, que necesita a la naturaleza - recursos naturales y trabajo- barata. Por esta razón, su lógica y sus modalidades de gobierno, que incluyen el uso sistemático de la violencia y el

\footnotetext{
${ }^{24}$ Ibídem, p. 15.

25 GROSFOGUEL, Ramón, "La actualidad del pensamiento de Césaire: redefinición del sistema-mundo y producción de utopía desde la diferencia colonial" en CESAIRÉ, Aime, Discurso..., op.cit., p. 148.

26 MEZZADRA, Sandra y RAHOLA, Federico, "La condición postcolonial. Unas notas sobre la cualidad del tiempo histórico en el presente global" en MEZZADRA, Sandro (coord.), Estudios..., op.cit., p. 265.

27 WALLERSTEIN, Immanuel, El moderno sistema mundial. La agricultura capitalista y los orígenes de la economíamundo europea en el siglo XVI, Siglo XXI Editores, Madrid, 1979; WALLERSTEIN, Immanuel, El moderno sistema mundial II. El mercantilismo y la consolidación de la economía-mundo europea, 1600-1750, Siglo XXI Editores, Madrid, 1984.

${ }^{28}$ MOORE, Jason W., "El auge de la ecología-mundo capitalista (I). Las fronteras mercantiles en el auge y decadencia de la apropiación máxima" en Laberinto, No 38, 2013, pp. 9-26; MOORE, Jason W., "El auge de la ecología-mundo capitalista (II). Las fronteras mercantiles en el auge y decadencia de la apropiación máxima" en Laberinto, No 39, 2013, pp. 21-29.

29 MARX, Karl, El capital..., op.cit.

30 HARVEY, David, El nuevo imperialismo, Akal, Madrid, 2004.

31 MOORE, Jason W., "El auge de la ecología-mundo capitalista (I)..., op.cit., pp. 9-26; MOORE, Jason W., "El auge de la ecología-mundo capitalista (I)..., op.cit., pp. 21-29.
} 
despojo, "han mantenido un rol continuo y persistente en la amplia geografía histórica de la acumulación de capital hasta nuestros días"32. Las prácticas imperialistas y, por ende, coloniales, se actualizan en prácticas neoimperialistas y neocoloniales para abrir nuevas fronteras funcionales a la acumulación y a la reproducción cotidiana y de largo plazo de las relaciones sociales capitalistas. Por ejemplo, el reciente caso del fracking para buscar el gas de esquisto y la difusión de un modelo económico neoextractivista en América Latina ${ }^{33}$ y en otras áreas del mundo son emblemáticos de esta tensión, como muestra la constante presencia de las multinacionales europeas en las ex colonias para disfrutar de la extracción de metales y minerales.

\section{Relaciones postcoloniales}

La colonia no ha desaparecido desde el punto de vista de las relaciones de poder entre metrópolis y periferias, pero este tipo de relación se desarrolla en un mundo postcolonial, en el mundo construido después del fin de la larga experiencia de la colonización política, militar y administrativa. La persistencia de relaciones de poder asimétricas entre las áreas centrales occidentales y una gran parte de las ex áreas coloniales, así como entre las poblaciones de las dos áreas, se concretiza en un mundo que ya ha vivido las experiencias de liberación colonial. Se puede hablar de una sociedad mundial que vive una condición histórica postcolonial, que "denota [...] al mismo tiempo continuidades y discontinuidades, pero pone el énfasis en las nuevas modalidades y formas de las viejas prácticas colonialistas, no en un «más allá»34.

Por ejemplo, los migrantes participantes en la asamblea en el Grand ghetto manifestaban que las relaciones coloniales de desigualdad estructural entre los blancos y las poblaciones no blancas - así como, más en general, entre los humanos y los otros miembros de la naturaleza- no se han acabado y siguen siendo actuales, funcionando como un potente elemento de continuidad del pasado colonial. Sin embargo, estas relaciones se actualizan en un tiempo postcolonial -después de las victoriosas luchas anticoloniales-donde se reconoce a todo el mundo un largo conjunto de derechos humanos, que definen un verdadero régimen internacional ${ }^{35}$. La humanidad se encuentra en un mundo articulado, donde esclavitud y estatus sociales o raciales de inferioridad han sido formalmente abrogados, pero algunas modalidades de gobierno de la colonia, como el control de la movilidad espacial, la división de la población en diferentes categorías con derechos y posibilidades diferenciadas, se han difundido de manera generalizada, también en la metrópoli. La sociedad postcolonial sigue estando basada estructuralmente en la desigualdad social, espacial y racial pese a ser una sociedad de igualdad formal.

Al mismo tiempo, la sociedad postcolonial se construye tras las luchas anticoloniales, que también han sido luchas populares y se han desarrollado conjuntamente con otros

\footnotetext{
${ }^{32}$ COMPOSTO, Claudia y PÉREZ ROIG, Diego, "Presentación. Trazos de sangre y fuego: ¿continuidad de la acumulación originaria en nuestra época?" en Theomai, No 26, 2012, p. 19.

33 DELGADO, Gian Carlo (coord.), Ecología política del extractivismo en América Latina: casos de resistencia y justicia socio-ambiental, CLACSO, Buenos Aires, 2013.

34 MEZZADRA, Sandro, "Introducción" en MEZZADRA, Sandro (coord.), Estudios postcoloniales..., op.cit., p. 13.

35 SASSEN, Saskia, Territorio, autoridad y derechos. De los ensamblajes medievales a los ensamblajes globales, Katz, Buenos Aires, 2010.
} 
movimientos antisistémicos, especialmente el movimiento obrero, feminista y estudiantili6. En consecuencia, sus miembros saben que las condiciones de desigualdad van aparejadas a las condiciones de conflicto y subversión. Esto es coherente con el hecho de que donde existen relaciones de poder también hay relaciones de resistencia: "donde hay poder hay resistencia, y no obstante -o mejor: por lo mismo-, ésta nunca está en posición de exterioridad respecto del poder" ${ }^{\prime \prime 3}$. En síntesis, se puede decir que la herencia colonial se manifiesta en la actualización de viejos dispositivos de dominación y en el saber históricamente fundado de que las relaciones de poder son delimitadas en el tiempo y que la historia de las relaciones sociales entre los dominantes y los subalternos siempre puede cambiar dado que esto ya ha ocurrido.

La herencia de las relaciones coloniales de poder es fuerte en el mundo postcolonial, contribuyendo a constituir las relaciones geopolíticas -en este sentido, es suficiente pensar en las relaciones asimétricas de muchas ex colonias independientes con los estados ex colonizadores- así como las relaciones entre los miembros de las ex sociedades colonizadas y colonizadoras. En este apartado, se presentan de manera más precisa algunas características de las relaciones coloniales que se han difundido y siguen activas en el mundo postcolonial.

Una parte de la literatura sobre el proceso de colonización, la construcción de la colonialidad y la postcolonialidad converge en el reconocimiento de que la característica fundamental de la colonia en la modernidad capitalista ha sido la de la separación jerárquica entre grupos diferentes de población. Se puede hablar de un principio constitutivo y de organización de la sociedad. Ello se ha articulado materialmente a través de la implementación de prácticas de confinamiento y reclusión, que han compartimentado a la población en grupos sociales y raciales tendencialmente homogéneos. De manera analítica, el paradigma políticosocial de la separación jerárquica ha implicado tres modalidades combinadas: en primer lugar, el confinamiento; en segundo lugar, la homogeneización; y en tercer lugar, la raza.

La primera modalidad ha sido indicada, entre otros, por Sandro Mezzadra y Federico Rahola, que han reconocido la reclusión y, esto es, el confinamiento como "la verdadera clave epistémica del proyecto de explotación colonial de Occidente y de la resistencia contra él" ${ }^{\prime 38}$. La multiplicación y la difusión de mecanismos de confinamiento - simbólico, espacial y social- y la continua producción de confines territoriales han sido funcionales a la producción de las jerarquías sociales y raciales ${ }^{39}$. La puesta en marcha por parte de las agencias públicas -normalmente manifestación directa o indirecta del estado o de alianzas interestatalesde acciones concretas y de dispositivos simbólicos dirigidos a controlar las áreas sociales, espaciales y racial-nacionales subalternas y desfavorecidas ha producido y reproducido la clasificación de la población en grupos distintos, desiguales y potencialmente en conflicto entre ellos.

${ }^{36}$ ARRIGHI, Giovanni et.al., Movimientos Antisistémicos, Akal, Madrid, 1999.

37 FOUCAULT, Michel, Historia de la sexualidad. La voluntad de saber, Vol. 1, Siglo Veintiuno Editores, Buenos Aires, 1998, p. 57.

38 MEZZADRA, Sandra y RAHOLA, Federico, "La condición postcolonial. Unas notas sobre la cualidad del tiempo histórico en el presente global" en MEZZADRA, Sandro (coord.), Estudios postcoloniales..., op.cit., p. 263.

39 MEZZADRA, Sandro y NEILSON, Brett, Border as method, or, the multiplication of labor, Duke University Press, Duhram y Londres, 2013. 
Entre estas acciones también se encuentra la producción de nuevas clases peligrosas, que normalmente incluyen a las poblaciones migrantes. Las personas migrantes han sido el objeto de la organización de herramientas administrativas o policiales, como los Centros de Identificación y Expulsión o las acciones coordinadas a nivel europeo de control y expulsión de los sin papeles, como la operación Mos Maiorum en el $2014^{40}$, y de herramientas militares de control de la movilidad de los seres humanos. Los migrantes se han convertido en el objetivo privilegiado de una guerra organizada por las grandes potencias mundiales, como por ejemplo a lo largo de la frontera entre México y Estados Unidos, a través del muro fronterizo entre Tijuana y San Diego, o en el área del Mediterráneo, por ejemplo a través de las barreras entre Marruecos y España y Grecia y Turquía o las operaciones de las agencias europeas para el control de las fronteras exteriores ${ }^{41}$.

La segunda modalidad, la de la homogeneización, viene reflejada de manera muy clara en la definición de colonización propuesta por la socióloga india Chandra Mohanty: un proceso que "en casi todos los casos implica una relación de dominación estructural y una supresión, muchas veces violenta, de la heterogeneidad del sujeto o sujetos en cuestión"42. La relación colonial trivializa y homogeneiza la realidad social y cultural, tendiendo a construir un espacio social liso y sin obstáculos.

La misma operación de homogeneización ha sido puesta en marcha por las narraciones coloniales dominantes en el caso de las mujeres del Tercer Mundo, pero también por una parte de la crítica postcolonial, especialmente la crítica del feminismo liberal y occidental. El sistema ha construido un sujeto femenino sin subjetividad y precisamente identificable. Las mujeres del Tercer Mundo se han convertido en la "víctimas par excellence"43 tanto por la ideología imperial, por un lado, como por las formas locales y extranjeras - coloniales- de patriarcado, por el otro. Según esta construcción teórica las mujeres son un sujeto homogéneamente marginal y subyugado, sin diferencias internas, $y$, sobre todo, con un destino predefinido y una colocación precisa, no modificable, en la geografía del poder postcolonial: un sujeto destinado a estar colocado en el margen. La homogeneidad reduce la realidad y, así, las diferentes modalidades de acción y expresión de los diferentes sujetos sociales.

La tercera modalidad, la de la raza, es central en muchos estudios críticos de las relaciones coloniales. Entre ellos, el análisis de Aníbal Quijano identifica la colonialidad como un elemento constitutivo del patrón del poder capitalista, basado -como ya reconoció William

40 STATEWATCH, Final report on Joint Operation "MOS MAIORUM", 2015:

http://www.statewatch.org/news/2015/jan/eu-council-2015-01-22-05474-mos-maiorum-final-report.pdf [Consultado el 11 de abril de 2017].

${ }^{41}$ BIGO, Didier y GUILD, Elspeth, "The Transformation of European Border Controls" en RYAN, Bernard y MITSILEGAS, Valsamis (coord.), Extraterritorial Immigration Control. Legal Challenges, Brill, Leiden, 2010, pp. 257-279; RIGO, Enrica, Europa di Confine. Trasformazioni della cittadinanza nell'Unione allargata, Meltemi, Roma, 2007.

42 MOHANTY, Chandra, "Bajo los ojos de Occidente. Academia Feminista y discurso colonial" en SUÁREZ NAVAZ, Liliana y HERNÁNDEZ, ROSALVA (coord.), Descolonizando el Feminismo. Teorías y Prácticas desde los Márgenes, Cátedra, Madrid, 2008, p. 114.

43 GANDHI, Leela, Postcolonial Theory. A Critical Introduction, Columbia University Press, Nueva York, 1998, p. 83. 
Edward Du Bois ${ }^{44}$ a través del concepto de la línea del color- en la clasificación jerárquica de las razas. Para Quijano, la colonialidad "se funda en la imposición de una clasificación racial/ étnica de la población del mundo como piedra angular de dicho patrón de poder y opera en cada uno de los planos, ámbitos y dimensiones, materiales y subjetivas, de la existencia social cotidiana y a escala societal" 45 .

En este sentido, la raza y los discursos raciales y racistas son una de las herencias más profundas de la colonización y de las relaciones coloniales. La referencia a la raza permite naturalizar las jerarquías entre los humanos clasificados en grupos homogéneos y dicotómicos -blancos y negros, blancos y mestizos- y, con ello, naturalizar todas las relaciones de poder vigentes -entre hombres y mujeres o empresarios y trabajadores, por ejemplo-. Los discursos coloniales de la raza han favorecido la construcción de una modalidad de pensamiento fundada en la separación jerárquica naturalizada de los grupos humanos que ha ido más allá del largo periodo colonial, convirtiéndose en un archivo de retóricas también utilizadas en el tiempo y en las relaciones postcoloniales. Consecuentemente, siguiendo los análisis propuestos por de Castro-Gómez y Grosfoguel ${ }^{46}$, se reconoce que "gran parte de los migrantes postcoloniales son objetos del racismo de la diferencia cultural, que desde los años setenta compone la nueva geocultura del sistema-mundo capitalista, y que refuerza y legitima las posiciones de subordinación de los inmigrantes periféricos en las metrópolis" ${ }^{\prime 7}$.

\section{El espacio político postcolonial y las migraciones}

La vida del conjunto de modalidades implicadas por el paradigma político-social de la separación jerárquica no se acabó con la liberación de las colonias: "el colonialismo no termina con el fin de la ocupación colonial"48. Esta consideración es evidente tanto en las relaciones entre los miembros de las ex colonias, especialmente en el caso de la construcción de los nuevos estados postcoloniales, como en las actitudes hacia los migrantes en las relaciones entre las poblaciones de las ex colonias y las poblaciones de las ex áreas colonizadoras.

Analizando el primer aspecto, se nota que las experiencias de edificación de los estados después de la colonia casi siempre han fracasado. Un caso paradigmático ha sido el caso argelino, analizado por el sociólogo Abdelmalek Sayad ${ }^{49}$, que ha evidenciado como la herencia colonial en las sociedades liberadas de la colonización se traduce en una falta de autonomía, porque las fuerzas políticas anti $-\mathrm{y}$ post- coloniales ponen en marcha procesos políticos e institucionales que repiten las antiguas $-y$ coloniales - modalidades de organización y ocupación del poder político, construyendo un estado basado en la represión y la violencia.

La misma contradicción se observa en el caso del gobierno y de la organización de las

${ }^{44}$ DU BOIS, William Edward Burghardt, "The Souls of Black Folks" en SUNDQUIST, Eric (coord.), The Oxford W. E. B. Du Bois Reader, Oxford University Press, Nueva York-Oxford, 1996, p. 107.

45 QUIJANO, Aníbal, "Colonialidad del poder y clasificación social" en CASTRO-GÓMEZ, Santiago y GROSFOGUEL, Ramón, El giro decolonial. Reflexiones para una diversidad epistémica más allá del capitalismo global, pp. 93126, Siglo del Hombre, Bogotá, 2007, p. 93.

${ }^{46}$ CASTRO-GÓMEZ, Santiago y GROSFOGUEL, Ramón (coord.), El giro decolonial..., op.cit.

${ }^{47} \mathrm{GIL}$, Sandra, "Abdelmalek Sayad. Una sociologia (de las migraciones) para la resistencia" en Empiria, No 19, 2010, p. 246.

48 GANDHI, Leela, Postcolonial Theory..., op.cit., p. 17.

49 SAYAD, Abdelmalek, Algeria: nazionalismo senza nazione, Mesogea, Messina, 2003. 
relaciones económicas y laborales, como ha sido puesto en evidencia de manera emblemática por el caso sudafricano de los disparos de la policía contra los trabajadores de la mina de Marikana el 16 de agosto de 2012, con 34 mineros asesinados ${ }^{50}$.

Sayad ${ }^{51}$ ha explicado que las migraciones se pueden entender solo estudiando de manera conjunta las emigraciones y las inmigraciones, los lugares de salida y los lugares de llegada. Al mismo tiempo, su investigación ha puesto en evidencia que las migraciones no se pueden entender fuera de las relaciones entre la historia de la colonización y la historia postcolonial. Por lo tanto, tomar en consideración el fracaso de muchos estados postcoloniales no es un hecho marginal para entender las nuevas migraciones postcoloniales. Este fracaso ha sido importante internamente en las sociedades de muchas ex colonias y también en la decisión de muchas personas de migrar y de construir un proyecto de vida a través de la dura experiencia de la migración.

Analizando el segundo aspecto, la comprensión de las migraciones postcoloniales es posible solo si se toma en consideración la permanencia del imaginario colonial y el papel que tiene en la organización de las modalidades de gobierno de las migraciones, contribuyendo a reproducir el espacio colonial más allá de las colonias, porque "todo el imaginario colonial que en buena parte es el éxito de la colonización (...) ha obsesionado y obsesiona, también más allá del verdadero período colonial, a todas las conciencias y a todos los espíritus que se refieren a ella, tanto hacia los colonizadores como hacia los colonizados, los de ayer así como sus descendientes" ${ }^{\prime \prime 2}$.

Para Sayad ${ }^{53}$, el mundo colonial es un mundo basado en la cólera, donde el mundo del colonizado se desarrolla justo al lado del mundo del colonizador, pero esos dos mundos no se enredan. Sayad reconoce la estructura de este mundo basada en el principio de la separación en el contexto postcolonial. Esto es el aspecto más evidente del enlace entre la experiencia colonial y la experiencia de la migración: la permanencia de relaciones similares de poder material y simbólico. La migración se pone en continuidad con la colonización y esta relación se observa en las atribuciones simbólicas, las jerarquías políticas y la colocación en las relaciones de producción que separan migrantes y población local.

El principio colonial de la separación está fortalecido por la distinción $-y$, por ende, la separación- institucional y epistemológica entre los nacionales y los no-nacionales determinada y reproducida por el pensamiento, y las prácticas consecuentes, de estado ${ }^{54}$.

Y ¿cuál es la práctica que pone en evidencia la continua presencia del principio de separación? Esta práctica es la del estado que puede expulsar a los extranjeros. El estado se constituye a través de la separación entre los que pertenecen y los que no pertenecen y,

${ }^{50}$ ALEXANDER, Peter et.al., Marikana: Voices from South Africa's Mining Massacre, Jacana, Johannesburg, 2013.

51 SAYAD, Abdelmalek, La doble ausencia. De las ilusiones del emigrado, a los padecimientos del inmigrado, Anthropos, Barcelona, 2010.

52 SAYAD, Abdelmalek, Algeria..., op.cit., pp. 36-37.

${ }^{53}$ SAYAD, Abdelmalek, "Colonialismo e migrações. Entrevista concedida a Federico Neiburg" en Mana. Estudos de Antropologia Social, Vol. 2, No 1, 1996, pp. 155-170.

${ }^{54}$ SAYAD, Abdelmalek, La doble ausencia..., op.cit. 
consecuentemente, está constitutivamente caracterizado por el poder de la expulsión. Los migrantes, por ello, se caracterizan por la condición de expulsabilidad ${ }^{55}$ y, por lo tanto, de permanente provisionalidad.

\section{Hacia un mundo de heréticos}

El mundo colonial afecta al mundo postcolonial, aunque no lo predefine totalmente. Hay un espacio de acción, que es un espacio de acción política, orientada al cambio de las relaciones de poder vigentes. El historiador indio Ranahit Guha expuso que la negación de la palabra y de la posibilidad de ejercer la acción política legítima son dos condiciones que definen la condición de subalternidad, típicas de las situaciones coloniales ${ }^{56}$. La misma negación es reconocida por Sayad en el caso de la condición migrante como una condición ajena al estado y al orden nacional, porque el migrante es definido como fuera del espacio del estado. Sin embargo, Sandra Gil evidencia que "Abdelmalek Sayad insiste en la imperiosa necesidad de reflexionar sobre la relación entre migración, orden nacional y pensamiento de estado"57. De hecho, Sayad propone realizar una ruptura herética con la visión del mundo social fundada en los órdenes de la inmigración y de la emigración, porque "es necesaria una ruptura verdaderamente herética con esos dos órdenes para concebir y hacer concebible, para prever y hacer prever, para enunciar y anunciar de otro modo la relación posible entre uno y otro"58. Así como se ha hecho durante la lucha anticolonial, los que están a los márgenes del espacio público, también en alianza con otros grupos sociales, pueden tomar la palabra y transformarse en sujetos políticos, abriendo posibilidades a la construcción de nuevas relaciones entre migrantes y nacionales y, en general, entre migración y orden estatal e interestatal.

Los que ponen en cuestión la separación entre nacionales y no nacionales son los que Sayad ha definido los heréticos, porque rompen el orden político 'normal', normalizado y normalizador, del estado. De hecho, los heréticos piensan y se organizan en el espacio público para proponer y construir un orden nacional definitivamente postcolonial. Ellos adoptan una actitud conflictiva hacia una situación llegada al pasado colonial y proponen un diferente patrón de relaciones sociales, más allá de la colonia, de la colonialidad y del pensamiento de estado.

La lucha anticolonial ha enseñado que los confines - sociales, políticos, simbólicosno son definitivos y no son insuperables y que los sujetos fuera del espacio político pueden convertirse en los principales actores de transformación política y social. El análisis de Sayad, reconociendo a los migrantes como los sujetos que inmediatamente ponen en cuestión el orden nacional y la separación constitutiva entre nacionales y no nacionales, desafiando los confines -territoriales y también sociales y simbólicos- cotidianamente, habla de la necesidad y de la posibilidad de definir de forma nueva las características del espacio político

55 DOMENECH, Eduardo, "La visión estatal sobre las migraciones en la Argentina reciente. De la retórica de la exclusión a la retórica de la inclusión" en DOMENECH, Eduardo (coord.), Migración y política: el Estado interrogado. Procesos actuales en Argentina y Sudamérica, Universidad Nacional de Córdoba, Córdoba, 2009, pp. 21-69.

${ }^{56}$ GUHA, Ränahit, Las voces de la historia y otros estudios subalternos, Crítica, Barcelona, 2002.

${ }^{57}$ GIL, Sandra, "Abdelmalek Sayad. Una sociologia (de las migraciones) para la resistencia" en Empiria, No 19, 2010, p. 244.

58 SAYAD, Abdelmalek, "Estado, nación e inmigración. El orden nacional ante el desafío de la inmigración" en Apuntes de investigación, № 13, 2008, p. 111. 
postcolonial. Este último se articula en relación a los dos polos de la herencia colonial: el de la dominación, que reproduce el principio de separación jerárquica, y el de la liberación, que cuestiona este principio a través de la difusión de prácticas heréticas.

Las prácticas de liberación tienen un rasgo necesariamente conflictivo puesto que ponen en cuestión lógicas políticas y sociales consolidadas y, sobre todo, funcionales a la conservación del orden. Estas prácticas tienen una larga historia de referencia, no solo en la lucha anticolonial sino en la lucha antiracista. Por ejemplo, las diferentes experiencias y propuestas políticas de Du Bois ponen en evidencia que, desde su origen, la construcción de alianzas entre poblaciones de orígenes territoriales, nacionales y de colores de la piel diferentes han jugado un papel importante en la lucha contra el racismo. La historia del Movimiento del Niágara y de la Asociación Nacional para el Progreso de las Personas de Color, fundados por Du Bois, respectivamente, en 1905 y 1909, es la de la cooperación antiracista entre negros y grupos de blancos, de manera coherente con el análisis elaborado por el mismo Du Bois ${ }^{59}$ sobre las relaciones entre los trabajadores: "para Du Bois, el pobre blanco y el trabajo blanco están conectados con el destino de los trabajadores negros y su incapacidad -o la incapacidad de sus líderes de trabajo- de reconocer esto ha conducido a la degradación reciproca tanto del trabajo negro como del trabajo blanco"60.

Esto no significa que los que están marginados en el espacio público - los no blancos o los no nacionales - no puedan tomar la palabra de manera autónoma. Esto se ha verificado históricamente y sigue verificándose, por ejemplo en el movimiento en Francia de los así llamado beurs, los hijos de los inmigrantes del norte de África, en los años ochenta ${ }^{61}$, o en las luchas de los sin papeles en diferentes áreas del mundo62. A su vez, otras luchas de los migrantes en los lugares de trabajo "ghetizados", como en las áreas agrícolas en Italia63, España ${ }^{64}$ y Baja California ${ }^{65}$, también así lo demuestran.

Lo que se evidencia es que las alianzas entre sujetos diferentes desde el punto de vista racial y del estado son constitutivamente alternativas al principio de separación jerárquica que normalmente gobierna y regula las relaciones sociales, especialmente entre nacionales y migrantes. Esto es evidente sobre todo en las luchas y en los movimientos organizados para la

${ }^{59}$ DU BOIS, William Edward Burghardt, Black Reconstruction in America. An Essay Toward a History of the Part Which Black Folk Played in the Attempt to Reconstruct Democracy in America, 1860-1880, Harcourt Brace and Company, Nueva York, 1935.

60 TAYLOR, Keeanga-Yamahtta, "Review of Black Reconstruction in America 1860-1880: WEB DuBois" en International Socialist Review, No 57, 2008, p. 26.

61 BETTEGAY, Alainy y BOUBERKER, Ahmed, "Des Minguettes au Vaulxen-Velin. Fractures sociales et discours publics" en Les Temps Modernes, No 545-546, 1992, pp. 51-76; BOUAMAMA, Said, Dix ans de marche des Beurs. Chronique d'un mouvement avorté, Desclée de Brouwer, París, 1994.

62 SUÁREZ-NAVAZ, Liliana et.al., Las luchas de los sin papeles y la extensión de la ciudadanía. Perspectivas críticas desde Europa y Estados Unidos, Traficantes de Sueños, Madrid, 2007; VARELA, Amarela, "Migrant struggles for the right to have rights: three examples of social movements powered by migrants in New York, Paris and Barcelona" en Transfer: European Review of Labour and Research, Vol. 14, No 4, 2008, pp. 677-694.

63 BRIGATE DI SOLIDARIETÀ ATTIVA et.al., Sulla pelle viva..., op.cit.

${ }^{64}$ CARUSO, Francesco, La politica dei subalterni Organizzazione e lotte del bracciantato migrante nel Sud Europa, Deriveapprodi, Roma, 2015.

${ }^{65}$ RODRÍGUEZ-SANTOS, Bertha, "Jornaleros de San Quintín: Un grito desesperado que rebasó las fronteras" en Ojarasca-La Jornada, No 216, 2015: http://www.jornada.unam.mx/2015/04/11/ojarasca216.pdf [Consultado el 11 de abril de 2017]. 
defensa de un derecho, un bien o una condición social o laboral común. El caso más evidente en los años recientes ha sido el de las luchas por el derecho a la vivienda en diferentes ciudades del mundo66, en las que, como ocurrió, por ejemplo, en un barrio de Málaga caracterizado por un alto número de desahucios, "gente de todos los colores y religiones se convertía en protagonista de la resistencia vecinal, y coreaban todos los lemas" ${ }^{\prime 67}$.

Alianzas sociales entre personas y grupos de diferentes nacionalidades son prácticas anti-racistas, que tienen la capacidad de crear un espacio político herético, cuestionando el principio de separación, característico de las relaciones coloniales, entre nacionales y migrantes y, además, entre blancos y no blancos, y, con ello, las relaciones de poder vigentes.

Un rol importante igualmente es el de las estrategias autónomas de los migrantes. Algunas investigaciones han reconocido esta capacidad también en el caso de la agricultura. Por ejemplo, la mano de obra en los campos de Morelos y Sinaloa en México reduce la injerencia y la fuerza de los contratistas sobre las modalidades de empleo mediante su saber circular y, frente a la ausencia de la acción activa del estado y de los empresarios para garantizar su derecho a la vivienda, organiza una industria de la migración desde abajo, transformando de manera autónoma espacios aislados de vida en lugares de socialidad ${ }^{68}$. Los trabajadores organizan estrategias diversificadas de reproducción social, estructurando nuevos circuitos migratorios, proyectos familiares y procesos de inserción socioterritorial o fuga que les permiten proteger su fuerza de trabajo, su dignidad y sus capacidades de resistencia: en síntesis, sus vidas.

\section{Conclusiones}

En la visión del pensamiento postcolonial el sufijo "post" se refiere más a las consecuencias y a los efectos duraderos de la experiencia colonial que a un tiempo posterior a ella. Ese sufijo se refiere a una dimensión relacional más que cronológica $y$, sobre todo, no individua un tiempo histórico simplemente libre del colonialismo sino un tiempo afectado por la herencia colonial, tanto en las prácticas y las modalidades de gobierno colonial como en las insurgencias anticoloniales. La referencia a la condición postcolonial es, entonces, una crítica a la temporalidad linear europea, basada en la idea de las etapas del desarrollo y en una especie de "ley determinista de rectilinearidad y de «unilinearidad»"69 temporal de la modernidad ${ }^{70}$.

Esta crítica ayuda a leer la sociedad contemporánea más allá de las narrativas del

${ }^{66}$ AVALLONE, Gennaro, "El movimento por la vivienda como práctica antirracista. La perspectiva de la ciudad mestiza contra la ciudad hostil en Italia" en Revista Movimentos Sociais e Dinâmicas Espaciais, Vol. 5, No 1, 2016, pp. 205-224.

67 GONZÁLEZ, Leticia, "Corrala" en CARRETERO, José Luis, Tu casa no es tuya, es del banco. Resistencias y alternativas colectivas frente al colapso de la burbuja inmobiliaria, Queimada, Madrid, 2015, p. 164.

68 LARA, Sara et.al., Asentamientos de trabajadores migrantes en torno a enclaves de agricultura intensiva en México: nuevas formas de apropiación de espacios en disputa, en PEDREÑO, Andrés (coord.), De cadena..., op.cit., pp. 150-169.

${ }^{69}$ GRAMSCI, Antonio, Cuadernos de la cárcel, Era/Benemérita Universidad autónoma de Puebla, Puebla, 1999, p. 362.

70 HALL, Stuart, "¿Cuándo fue lo 'postcolonial'? Pensando en el límite" en HALL, Stuart, Sin garantías. Trayectorias y problemáticas en estudios culturales, Envión Editores-Instituto Pensar-IEP-Universidad Andina, Bogotá-LimaQuito, 2010, pp. 563-582. 
progreso capitalista y es particularmente útil para comprender la inserción de la mano de obra migrante en agricultura y como ella está gobernada y definida por la persistencia de formas de pensamiento y de actitudes coloniales orientadas a diferenciar de manera jerárquica las poblaciones.

Las migraciones tienen un carácter postcolonial puesto que se alimentan del doble significado del pasado colonial -el de la dominación y el de la liberación- desafiando a los dispositivos de control heredados y nuevos. De hecho, el fin de las administraciones coloniales no ha significado el inicio de un proceso de liberación definitivo de los mecanismos de separación jerárquica entre los miembros de la sociedad, sino una difusión de las fronteras, de los mecanismos de control y de las prácticas de clasificación de los diferentes grupos humanos. Las fronteras, lejos de reducirse, se han multiplicado, y difundido fuera de los (ex) espacios coloniales, potencialmente a lo largo de todo el mundo y contra la movilidad autónoma de los seres humanos.

Ya no hay una diferencia clara, precisa y geográficamente signada entre colonias y metrópolis $y$, en esta nueva realidad, el tiempo postcolonial hace posible la construcción de nuevas relaciones políticas y sociales, también a través de nuevas alianzas entre parte de la población local y parte de la población migrante: alianzas que, de manera sintética, se podían llamar mestizas, más allá del orden nacional de la separación, orientadas hacia un común horizonte de derechos y de respuestas a las necesidades sociales de las poblaciones.

\section{Bibliografía}

ARRIGHI, Giovanni, HOPKINS, Terence Y WALLERSTEIN, Immanuel, Movimientos Antisistémicos, Akal, Madrid, 1999.

ALEXANDER, Peter, MMOPE, Botsang, LEKGOWA, Thapelo, XEZWI, Bongani, SINWELL, Luke, Marikana: voices from south africa's mining massacre, Jacana, Johannesburg, 2013.

AVALLONE, Gennaro, "El movimento por la vivienda como práctica antirracista. La perspectiva de la ciudad mestiza contra la ciudad hostil en Italia" en Revista Movimentos Sociais e Dinâmicas Espaciais, Vol. 5, № 1, 2016, pp. 205-224.

AVALLONE, Gennaro, "Migraciones y agricultura en Europa del Sur: Emergencia de un nuevo proletariado internacional" en Migraciones Internacionales, Vol. 7, No 4, 2014, pp. 137-169.

BETTEGAY, Alainy y BOUBERKER, Ahmed, "Des Minguettes au Vaulxen-Velin. Fractures sociales et discours publics" en Les Temps Modernes, No 545-546, 1992, pp. 51-76.

BIGO, Didier y GUILD, Elspeth, "The Transformation of European Border Controls" en RYAN, Bernard y MITSILEGAS, Valsamis (coords.), Extraterritorial Immigration Control. Legal Challenges, Brill, Leiden, 2010, pp. 257-279.

BONANNO, Alessandro y BARBOSA CAVALCANTI, Josefa Salete, "Introduction" en BONANNO, Alessandro y BARBOSA CAVALCANTI, Josefa Salete (coords.), Labor Relations in Globalized Food, Emerald Group Publishing Limited, Bingley, 2014, pp. 13-49.

BOUAMAMA, Said, Dix ans de marche des Beurs. Chronique d'un mouvement avorté, Desclée de Brouwer, París, 1994.

BRIGATE DI SOLIDARIETÀ ATTIVA, SACCHETTO, Devi, NIGRO, Gianluca, PERROTTA, Mimmo, SAGNET, Yvan, Sulla pelle viva. Nardó: la lotta autorganizzata dei braccianti immigrati, DeriveApprodi, Roma, 2012.

BUCK-MORSS, Susan, Hegel y Haiti. La dialéctica amo-esclavo: una interpretación revolucionaria, Grupo Editorial Norma, Buenos Aires, 2005.

CARUSO, Francesco, La politica dei subalterni Organizzazione e lotte del bracciantato migrante nel Sud Europa, Deriveapprodi, Roma, 2015.

CASTRACANI, Lucio, "Étranger(s) au travail. Notes ethnographiques" en Altérités, Vol. 9, № 1, 2016, pp. 43-55.

CASTRO-GÓMEZ, Santiago y GROSFOGUEL, Ramón (coord.), El giro decolonial. Reflexiones para una 
diversidad epistémica más allá del capitalismo global, Siglo del Hombre, Bogotá, 2007.

CÉSAIRE, Aimé, Discurso sobre el colonialismo, Akal, Madrid, 2006.

COLLOCA, Carlo y CORRADO, Alessandra (coords.), La globalizzazione delle campagne. Migranti e società rurali nel Sud Italia, FrancoAngelli, Milán, 2012.

COMPOSTO, Claudia y PÉREZ ROIG, Diego, "Presentación. Trazos de sangre y fuego: ¿continuidad de la acumulación originaria en nuestra época?" en Theomai, № 26, 2012, pp. 1-22.

DE CASTRO, Carlos, "La desdemocratización de las relaciones laborales en los enclaves globales de producción agrícola" en PEDREÑO CÁNOVAS, Andrés (coord.), De cadenas, migrantes y jornaleros, Talasa, Madrid, 2014, pp. 59-77.

DELGADO, Gian Carlo (coord.), Ecología política del extractivismo en América Latina: casos de resistencia y justicia socio-ambiental, CLACSO, Buenos Aires, 2013.

DOMENECH, Eduardo, "La visión estatal sobre las migraciones en la Argentina reciente. De la retórica de la exclusión a la retórica de la inclusión" en DOMENECH, Eduardo (coord.), Migración y política: el Estado interrogado. Procesos actuales en Argentina y Sudamérica, Universidad Nacional de Córdoba, Córdoba, 2009, pp. 21-69.

DU BOIS, William Edward Burghardt, Black Reconstruction in America. An Essay Toward a History of the Part Which Black Folk Played in the Attempt to Reconstruct Democracy in America, 1860-1880, Harcourt Brace and Company, Nueva York, 1935.

DU BOIS, William Edward Burghardt, "The Souls of Black Folks" en Sundquist, Eric (coord.), The Oxford W. E. B. Du Bois Reader, Oxford University Press, Nueva York-Oxford, 1996.

DUSSEL, Enrique, "Eurocentrismo y modernidad (Introducción a las lecturas de Frankfurt)" en MIGNOLO, Walter (coord.), Capitalismo y geopolítica del conocimiento: el eurocentrismo y la filosofía de la liberación en el debate intelectual contemporáneo, Signo, Buenos Aires, 2001, pp. 57-70.

FRANCES, Jennifer, BARRIENTOS, Stephanie y ROGALY, Ben, Temporary workers in UK agriculture and horticulture: a study of employment practices in the agriculture and horticulture industries and co-located packhouse and primary food processing sectors, Precision Prospecting for the Department of Environment, Food and Rural Affairs (DEFRA), Framlingham, 2005.

FOUCAULT, Michel, Historia de la sexualidad. La voluntad de saber, Vol. 1, Siglo Veintiuno Editores, Buenos Aires, 1998.

GANDHI, Leela, Postcolonial Theory. A Critical Introduction, Columbia University Press, Nueva York, 1998.

GARCÍA, Martha y DÉCOSSE, Frédéric, "Agricultura intensiva y políticas de migración laboral: Jornaleros centroamericanos en México y marroquíes en Francia" en Migración y desarrollo, Vol. 12, No 23, 2014, pp. 41-67.

GERTEL, Jörg y SIPPEL, Sarah Ruth (coord.), Seasonal Workers in Mediterranean Agriculture. The social costs of eating fresh, Routledge, Londres, 2014.

GIL, Sandra, "Abdelmalek Sayad. Una sociología (de las migraciones) para la resistencia" en Empiria, No 19, 2010, pp. 235-249.

Gramsci, Antonio, Cuadernos de la cárcel, Era/Benemérita Universidad autónoma de Puebla, Puebla, 1999.

GONZÁLEZ, Leticia, "Corrala" en CARRETERO, José Luis, Tu casa no es tuya, es del banco. Resistencias y alternativas colectivas frente al colapso de la burbuja inmobiliaria, Queimada, Madrid, 2015, pp. 159-176.

GROSFOGUEL, Ramón, "La actualidad del pensamiento de Césaire: redefinición del sistema-mundo y producción de utopía desde la diferencia colonial" en CESAIRÉ, Aime, Discurso sobre el colonialismo, Akal, Madrid, 2006, pp. 147-172.

GUHA, Ränahit, Las voces de la historia y otros estudios subalternos, Crítica, Barcelona, 2002.

HALL, Stuart, "¿Cuándo fue lo 'postcolonial'? Pensando en el límite" en HALL, Stuart, Sin garantías. Trayectorias y problemáticas en estudios culturales, Envión Editores-Instituto Pensar-IEPUniversidad Andina, Bogotá-Lima-Quito, 2010, pp. 563-582.

HARVEY, David, El nuevo imperialismo, Akal, Madrid, 2004.

JAMES, Cyril Lionel Robert, Los jacobinos negros. Toussaint L'Ouverture y la Revolución de Haití, Fondo de Cultura Económica, México, 2003.

KASIMIS, Charalambos, PAPADOPOULOS, Apostolos y ZOGRAFAKIS, Stavros, "The Precarious Status of Migrant Labour in Greece: Evidence from Rural Areas" en DELLA PORTA Donatella, SILVASTI, Tiina HÄNNINEN, Sakari y SIISIÄINEN, Martti (coord.), The New Social Division Making and Unmaking Precariousness, Palgrave Macmillan, Basingstoke, 2015, pp. 101-120.

LARA, Sara, SÁNCHEZ, Kim y SALDAÑA, Adriana, "Asentamientos de trabajadores migrantes en torno a enclaves de agricultura intensiva en México: nuevas formas de apropiación de espacios en disputa" en PEDREÑO, Andrés (coord.), De cadena, migrantes y jornaleros. Los territorios rurales en las cadenas globales agroalimentarias, Talasa, Madrid 2014, pp. 150-169.

MARTIN, Philip, California Hired Farm Labor 1960-2010: Change and Continuity, University of California, 30 de abril de 2011, p. 5: https://migrationfiles.ucdavis.edu/uploads/cf/files/2011-may/martin- 
california-hired-farm-labor.pdf [Consultado el 23 de julio de 2017].

MARX, Karl, El capital. Volume 1, Siglo XXI, Buenos Aires, 2002.

MEZZADRA, Sandro, "Introducción" en MEZZADRA, Sandro (coord.), Estudios postcoloniales. Ensayos fundamentales, Traficantes de Sueños, Madrid, 2008, pp. 15-33.

MEZZADRA, Sandro y NEILSON, Brett, Border as method, or, the multiplication of labor, Duke University Press, Duhram y Londres, 2013.

MEZZADRA, Sandra y RAHOLA, Federico, "La condición postcolonial. Unas notas sobre la cualidad del tiempo histórico en el presente global" en MEZZADRA, Sandro (coord.), Estudios postcoloniales. Ensayos fundamentales, Traficantes de Sueños, Madrid, 2008, pp. 261-278, p. 263.

MOHANTY, Chandra, "Bajo los ojos de Occidente. Academia Feminista y discurso colonial" en SUÁREZ NAVAZ, Liliana y HERNÁNDEZ, Rosalva (coord.), Descolonizando el Feminismo. Teorías y Prácticas desde los Márgenes, Cátedra, Madrid, 2008, pp. 112-160.

MOORE, Jason W., Capitalism in the web of life: ecology and the accumulation of capital, Verso, Londres y Nueva York, 2015.

MOORE, Jason W., "El auge de la ecología-mundo capitalista (I). Las fronteras mercantiles en el auge y decadencia de la apropiación máxima" en Laberinto, No 38, 2013, pp. 9-26.

MOORE, Jason W., "El auge de la ecología-mundo capitalista (II). Las fronteras mercantiles en el auge y decadencia de la apropiación máxima" en Laberinto, No 39, 2013, pp. 21-29.

MOORE, Jason W., "'This lofty mountain of silver could conquer the whole world': Potosí and the political ecology of underdevelopment, 1545-1800" en The Journal of Philosophical Economics, Vol. IV, No 1,2010 , pp. 58-103.

ORTIZ, Celso, "Migración, precariedad y sindicalización en la agricultura globalizada" en La Jornada del campo, No 94, 2015: http://www.jornada.unam.mx/2015/07/18/cam-migracion.html [Consultado el 11 de abril de 2017].

PAPADOPOULOS, Apostolos y FRATSEA, Loukia-Maria, "Migrant labour and intensive production in Greece" en CORRADO, Alessandra, DE CASTRO, Carlos y PERROTTA, Domenico (coord.), Migration and agriculture. Mobility and change in the Mediterranean area, Routledge, Nueva York-Londres, 2016, pp. 128-143.

PEDREÑO CÁNOVAS, Andrés, "Encadenados a fetiches. Del enfoque de las cadenas de mercancías a la sosteniblidad social de los enclaves de producción de la 'uva global'" en PEDREÑO CÁNOVAS, Andrés (coord.), De cadenas, migrantes y jornaleros, Talasa, Madrid, 2014, pp. 13-37.

PERROTTA, Domenico y SACCHETTO, Devi, "Migrant Farmworkers in Southern Italy: Ghettoes, caporalato and collective action" en Workers of the World: International Journal on Strikes and Social Conflicts, Vol. 1, No 5, 2014, pp. 75-98.

QUIJANO, Anibal, "Colonialidad del poder y clasificación social" en CASTRO-GÓMEZ, Santiago y GROSFOGUEL, Ramón, El giro decolonial. Reflexiones para una diversidad epistémica más allá del capitalismo global, Siglo del Hombre, Bogotá, 2007, pp. 93-126.

RIGO, Enrica, Europa di Confine. Trasformazioni della cittadinanza nell'Unione allargata, Meltemi, Roma, 2007.

RODRÍGUEZ-SANTOS, Bertha, "Jornaleros de San Quintín: Un grito desesperado que rebasó las fronteras" en Ojarasca-La Jornada, No 216, 2015: http://www.jornada.unam.mx/2015/04/11/ ojarasca216.pdf [Consultado el 11 de abril de 2017].

ROGALY, Ben, "Migrant Workers in the Ilo's 'Global Alliance against Forced Labour'. Report: A Critical Appraisal" en Third World Quarterly, Vol. 29, No 7, 2008, pp. 1431-1447.

SASSEN, Saskia, Territorio, autoridad y derechos. De los ensamblajes medievales a los ensamblajes globales, Katz, Buenos Aires, 2010.

SAYAD, Abdelmalek, La doble ausencia. De las ilusiones del emigrado, a los padecimientos del inmigrado, Anthropos, Barcelona, 2010.

SAYAD, Abdelmalek, "Estado, nación e inmigración. El orden nacional ante el desafío de la inmigración" en Apuntes de investigación, No 13, 2008, pp. 101-116.

SAYAD, Abdelmalek, Algeria: nazionalismo senza nazione, Mesogea, Messina, 2003.

SAYAD, Abdelmalek, "Colonialismo e migrações. Entrevista concedida a Federico Neiburg" en Mana. Estudos de Antropologia Social, Vol. 2, No 1, 1996, pp. 155-170.

STATEWATCH, Final report on Joint Operation "MOS MAIORUM", 2015: http://www.statewatch.org/ news/2015/jan/eu-council-2015-01-22-05474-mos-maiorum-final-report.pdf [Consultado el 11 de abril de 2017].

STOLER, Ann Laura y COOPER, Frederick, "Between Metropole and Colony. Rethinking a Research Agenda" en STOLER, Ann Laura y COOPER, Frederick (coord.), Transitions of Empire. Colonial Cultures in a Bourgeois World, University of California Press, Berkeley, 1997, pp. 1-56.

SUÁREZ-NAVAZ, Liliana, PAREJA, Raquel Macià y MORENO, Ángela, Las luchas de los sin papeles y la extensión de la ciudadanía. Perspectivas críticas desde Europa y Estados Unidos, Traficantes de Sueños, Madrid, 2007.

TAYLOR, Keeanga-Yamahtta, "Review of Black Reconstruction in America 1860-1880: WEB DuBois" en 
International Socialist Review, No 57, 2008, pp. 25-30.

VARELA, Amarela, "Migrant struggles for the right to have rights: three examples of social movements powered by migrants in New York, Paris and Barcelona" en Transfer: European Review of Labour and Research, Vol. 14, No 4, 2008, pp. 677-694.

WALLERSTEIN, Immanuel, El moderno sistema mundial. La agricultura capitalista y los orígenes de la economía-mundo europea en el siglo XVI, Siglo XXI Editores, Madrid, 1979.

WALLERSTEIN, Immanuel, El moderno sistema mundial II. El mercantilismo y la consolidación de la economía-mundo europea, 1600-1750, Siglo XXI Editores, Madrid, 1984.

WRIGHT, Gwendolyn, "Tradition in the Service of Modernity: Architecture and Urbanism in French Colonial Policy" en Journal of Modern History, No 59, 1987, pp. 291-316. 


\section{RELACIONES INTERNACIONALES}

Revista académica cuatrimestral de publicación electrónica Grupo de Estudios de Relaciones Internacionales (GERI) Universidad Autónoma de Madrid, España

www.relacionesinternacionales.info

ISSN 1699 - 3950

ff facebook.com/RelacionesInternacionales

twitter.com/RRInternacional 